\title{
Superior thyroid artery pseudoaneurysm as a complication of transesophageal echocardiography
}

\begin{abstract}
The pseudo-aneurysm is a hematoma of post-traumatic origin, and capsulated button which is in communication with the lumen of the artery of relevance. Singular occurrence in the district ENT, if not recognized early the pseudo-aneurysm can result in dramatic events such cataclysmic bleeding or acute occlusion of the upper airway. In literature there are outstanding references to the pseudo-aneurysm of the superior thyroid (ATS). We present a rare case of pseudo-aneurysm occurred after the ATS trans-esophageal echocardiography (TEE) and external cardioversion.
\end{abstract}

Keywords: pseuduoaneurysm, ent complication, endovascular coil embolization
Volume 2 Issue 2 - 2015

\section{Frank Rikki Canevari, Stefania Galla, Emma Aina, Raffaele Sorrentino}

Department of Otorhinolaryngology, Azieneda ospedaliera SS Antonio, Italy

\author{
Correspondence: Frank Rikki Canevari, Department of \\ Otorhinolaryngology, Azieneda ospedaliera SS Antonio, Biagio \\ e Cesare Arrigo,Via Venezia 332000 Alessandtria, Italy, Tel \\ +39333 |49|318,Email c.rikki@alice.it
}

Received: January 12, 2015 | Published: January 23, 2015
Abbreviations: STA, pseudo-aneurysm of the superior thyroid; TEE, trans-esophageal echocardiography; FNAC, fine needle aspiration biopsy

\section{Introduction}

Pseudoaneurysm is a pulsating hematoma that results from a tangential injury to an arterial vessel wall and could represent a complication after trauma. In head and neck region pseudoaneurysm is rare but could have catastrophic consequences. For this reason it must be recognized to prevent such events as hemorrhage or acute occlusion of the airway. In literature a pseudoaneurysm arising from superior thyroid artery (STA) has been reported only in tree cases, in one case after ultrasonographically guided chemical parathyriodectomy, one after radiotherapy for hypopharyngeal cancer and the last after fine needle aspiration biopsy (FNAC)of thyroid nodule. We report a case of STA after transesophageal ecocardiography guided cardioversion.

\section{Case presentation}

A 62-year-old man was admitted in our Otolaryngology Department of presenting sore throat, disphagia and mild dyspnea after a transesophageal ecocardiography guided cardioversion performed 3 days before. The endoscopic examination of upper aereodigestive tract showed a diffuse soft edema of arytenoids and pharyngolaryngeal tract without airway obstruction. A corticosteroid therapy was started and the symptoms quickly improved but two days later the patient presented suddenly a hemorrhage from the superior aereodigestive tract. The endoscopic examination revealed a swelling of the left lateral wall of the hypopharynx and a hemorrhage from the apex of the swelling witch stopped spontaneous after few minutes. A CT scan of neck revealed an active arterial bleeding with pseudoaneurysmal dilatation of $1,2 \mathrm{~cm}$ of diameter arising from a fine branch of external carotid artery and a well defined homogeneously enhancing mass, $9.5 \times 3.2 \mathrm{~cm}$ diameter in left neck spaces extending superiorly to parapharyngeal spaces, inferiorly to hypopharynx displacing hyoid bone, thyroid cartilage, posterior to crycoid cartilage displacing cervical esophagus and laterally to subcutaneous tissue displacing sternocleidomastoid muscle (Figure 1). The mass was suggestive for hematoma. The angiography revealed a pseudoaneurysm with active bleeding of the terminal tract of superior thyroid artery (STA) (Figure 2). An endovascular procedure was performed with a superselctive microcateter and a endovascular occlusion by coil embolizzation of STA with exclusion of the pseudoaneurysm demonstrated at the end of the procedure (Figure 3). The symptoms resolved after one day from the endovascular procedure. The patient was discharged after two days without evidence of bleeding and an endoscopic control after 4 weeks revealed a disappearance of swelling.

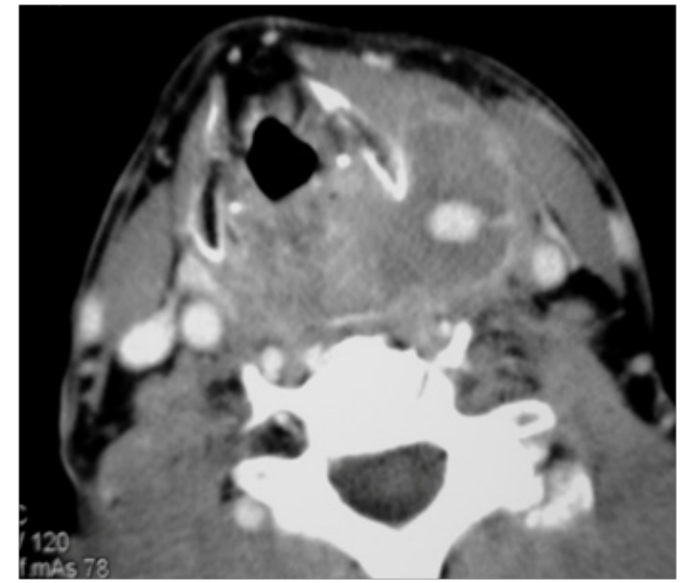

Figure I Axial CT scan illustrating pseudoaneurysmal dilatation arising from a fine branch of external carotid artery.

\section{Discussion}

Pseudoaneurysm, also called "false aneurysm", is an harmatoma contained by adventitia or perivascular tissue communicating with an arterial vessel that results from a tangential injury to the vessel. ${ }^{1,2}$ Most often it is secondary to blunt and penetrating trauma ${ }^{1,3,4}$ or surgery ${ }^{5-7}$ and is a well-known as iatrogenic complication of vascular catheterization $^{8,9}$ or percutaneous biopsy of solid organs. ${ }^{10}$

Pseudoaneurysm of head and neck are quite rare events but could lead to catastrophic complications. Clinical manifestations can include pulsating mass in the neck, dysphagia, dyspnea, pain in the neck, bleeding from oral cavity and cerebrovascular symptoms. The differential diagnosis of an expansive cervical mass includes bronchial cleft cyst, neck abscess and cervical adenitis. Other cervical masses such as tumors, lymphoadenopathy or neurinomas can be excluded by 
timing of growing of the mass. Many imaging techniques are available to differentiate the diagnosis. Color Doppler ultrasound is an excellent technique to detect the presence of an aneurismal sac and the presence of an active bleeding. MRI and CT scan of the neck give information of the involment of neck anatomical structures. Angiography is the best technique to investigate the morphology of the vessel involved and the source of the aneurismal sac.

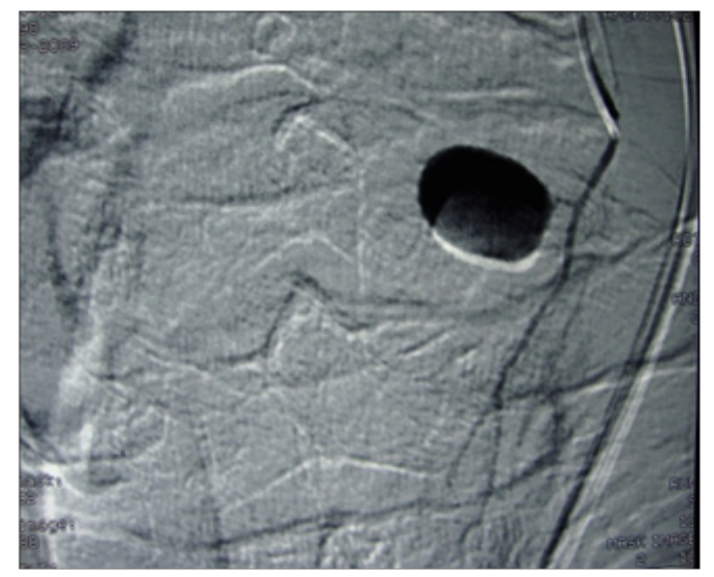

Figure 2 Angiography illustrating the pseudoaneurysm with active bleeding of the terminal tract of superior thyroid artery (STA).

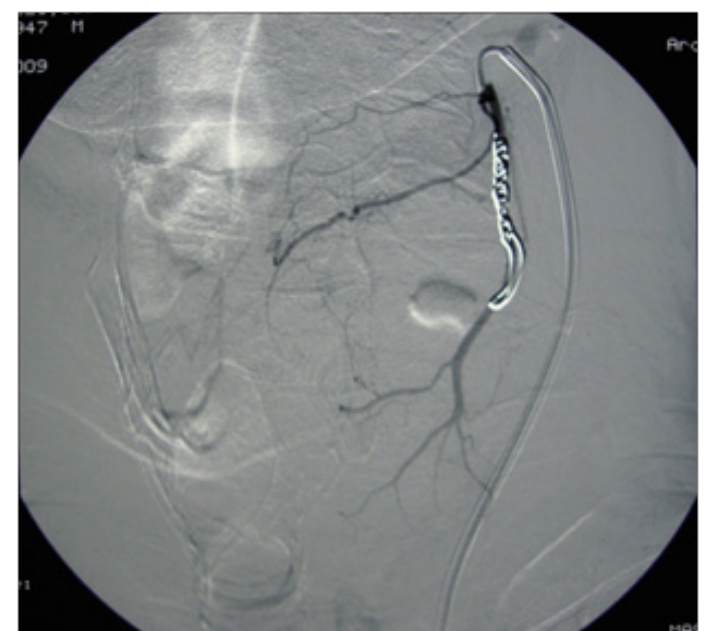

Figure 3 Angiography illustrating endovascular occlusion by coil embolization.

Management options for pseudoaneurysm include conservative measures with or without sonographically guided compression therapy, ${ }_{10,11}$ surgical repair, ${ }^{12}$ transarterial coil embolization ${ }^{13}$ and percutaneous thrombin or alcohol injection. ${ }^{14}$ There is no standard treatment protocol because of the rarity of neck pseudoaneurysm and the adequate treatment should be determined on case-by-case basis.

A fast development of an expanding mass under the angle of the mandible or in lateral pharyngeal wall after surgical procedure of the neck, percutaneous biopsy and neck trauma should always raise the suspect of an extra cranial arterial pseudoaneurysm.

In our knowledge only three cases of pseudoaneurysm of superior thyroid artery have been reported previously. The first case was a pseudoaneurysm occurred after ultrasonographically guided chemical parathyriodectomy. ${ }^{15}$ The diagnosis was made by angiography and treated by selective coil embolization. The second case reported a pseudoaneurysm occurred in a patient with hypopharyngeal squamous cell carcinoma during simultaneous radiotherapy and chemotherapy. ${ }^{13}$ The diagnosis was made by a CT scan and treated by selective coil embolization. The third case reported a pseudoaneurysm after ultrasonographically guided biopsy of a thyroid nodule. ${ }^{10}$ The diagnosis was made after ultrasonography and color Doppler examination and treated waiting spontaneous thrombosis.

In our patient the pseudoaneurysm occurred after a transesophageal echocardiography guided cardioversion (TEE-guided). The diagnosis was made by a computed tomography scan and treated by selective coil embolization. Transesophageal echocardiography guided cardioversion with short-term anticoagulation can be considered a safe and clinically effective for patients with atrial fibrillation. Complications reported after TEE-guided procedure includes bleeding events. We describe a unique case of superior thyroid artery pseudoaneurysm after TEE-guided.

Pseudoaneurysm in the neck are quite rare event but a rapid growing of an expanding mass in the neck after surgical procedure of the neck, percutaneous biopsy and neck trauma or transoral procedures should always raise the suspect of an extra cranial arterial pseudoaneurysm. A rapid growing mass in the neck can led to an airway acute obstruction and a fast diagnosis and treatment is required in order to prevent catastrophic consequences .In according with literature there are not protocols for diagnosis and treatment. In our opinion CT scan for differential diagnosis and visualization of anatomical structures involved and a transarterial coil embolization can be considered a safe and effective diagnostic and treatment procedures.

\section{Acknowledgments}

None.

\section{Conflicts of interest}

The authors declare that there are no conflicts of interest.

\section{References}

1. Krempl GA, Noorily AD. Pseudoaneurysm of the descending palatine artery presenting as epistaxis. Otoloaryngol Head Neck Surg. 1996;114(3):453-456.

2. Reiber ME, Burkey BB. Intracavernous carotid pseudoaneurysm after blunt trauma: case report and discussion. Head Neck. 1994;16(3):253-258.

3. Brown MF, Graham, Feliciano DV, et al. Carotid artery injuries. Am J Surg. 1982;144(6):748-753.

4. Han MH, Sung MW, Chang KH, et al. Traumatic pseudoaneurysm of the intracavernous ICA presenting with massive epistaxis: imaging, diagnosis and endovascular treatment. Laryngoscope. 1994;104(3Pt1):370-377.

5. Smelt JL, Alhamarneh O, Dyer JD, et al. Endovascular coiling of mycotic external carotid artery pseudoaneurysm following pharyngolaryngectomy with a free jejunal graft. J Laryngol otol. 2012;126(2):214-216.

6. Kras DE, Sawin RS, Sie KC. Pseudoaneurysm of the external carotid artery after tonsillectomy. A rare complication. Arch Otolaryngol Head Neck Surg. 1997;123(3):345-347.

7. Cockroft KM, Carew JF, Trost D, et al. Delayed epistaxis resulting from external carotid artery unjury requirering embolization: a rare complication of transphenoidal surgery: case report. Neurosurgery. 2000;47(1):236-239.

8. Kua JSW, Tan IKS. Airway obstruction following internal jugular vein 
cannulation. Anaesthesia. 1997:52(8):776-785.

9. Parry W, Dhillon R, Salahudeen A. Carotid pseudoaneurysm from inadvertent carotid artery catheterization for haemodialysis. Nephrol Dial Transplant. 1996;11(9):1853-1855.

10. Celik H, Yucel C, Oktar S, et al. Iatrogenic pseudoaneurysm of the superior thyroid artery: color Doppler ultrasonographic diagnosis and treatment approach. J Ultrasound Med. 2004;23(12):1675-1678.

11. Schaub F, Theiss W, Heinz M, et al. New aspect in ultrasound-guided compression repair of postcatheterization femoral artery injuries. Circulation. 1994;90(4):1861-1865.

12. Walker AT, Chaloupka JC, Putman CM, et al. Sentinal transoral hemorrhage from a pseudoaneyrism of internal maxillary artery: a complication of Ct-giuded biopsy of the masticator space. AJNR Am J Neuroradiol. 1996;17(2):377-381.

13. Ernemann U, Herrrmann C, Plontke S, et al. Pseudoaneurysm of the superior thyroid artery following radiotherapy for hypopharyngeal cancer. Ann Otol Rhinol Laryngol. 2003;112(2):188-190.

14. Mann GS, Heran MK. Percutaneous thrombin injection of a superficial temporal artery pseudoaneurysm. Pediatr Radiol. 2007;37(6):578-580.

15. Perona F, Barile A, Olivieri M, et al. Superior thyroid artery lesion after us-guided chemical parathyroidectomy: angiographic diagnosis and treatment by embolization. Cardiovasc Intervent Radiol. 1999;22(3):249-250. 